\title{
Low Voltage Thermo-mechanically Driven Monolithic Microgripper with Piezoresistive Feedback
}

\author{
Vladimir Stavrov ${ }^{1, *}$, Emil Tomerov ${ }^{1}$, Chavdar Hardalov ${ }^{2}$, Daniel Danchev ${ }^{3}$, \\ Kostadin Kostadinov ${ }^{3}$, Galina Stavreva ${ }^{1}$, Evstati Apostolov ${ }^{2,4}$, Assen Shulev ${ }^{3}$, \\ Anna Andonova ${ }^{2}$, and Mohammed Al-Wahab ${ }^{5}$ \\ ${ }^{1}$ AMG Technology Ltd., Industrial Zone Microelectronica, Botevgrad, Bulgaria \\ ${ }^{2}$ Sofia Technical University, Sofia, Bulgaria \\ ${ }^{3}$ Institute of Mechanics and Biomechanics, BAS, Sofia, Bulgaria \\ ${ }^{4}$ Micro Plus - Apostolov Ltd., Sofia Bulgaria \\ ${ }^{5}$ University Otto-von-Guiricke, Magdeburg, Germany \\ vs@amg-t.com
}

\begin{abstract}
Pick-and-place of micro/nano sized objects means handling of very tiny and very different in properties objects having specific behavior. Besides formal requirements of assembly processes, the tools for controllable manipulation with these objects should not affect the examined micro/nano environment, i.e. should be "small and passive" in any sense. Despite of the recent progress, most available micro-grippers are still suffering of high voltage power supply required, short lifetime, low detection sensitivity and high price.

Prototypes of a newly designed micro-gripper, having advantages over the existing analogues, have been developed, experimentally studied, and presented in this paper. The envisaged microgripper is of normally-closed type with thermo-mechanically driven actuator and piezoresistive arm-displacement feedback. The thermo actuator is placed between gripper's arms and consists of double-folded highly-doped compliant silicon beam. As low average voltage vs. arm displacement value as $1 \mathrm{~V} / \mu \mathrm{m}$, was experimentally measured.
\end{abstract}

Keywords: MEMS, micro-gripper, piezoresistive detection.

\section{Introduction}

Formal requirements and design trade-off for pick-and-place micro assembly for microgrippers are well described elsewhere [1]. One of the important characteristic features is to provide feedback during manipulation with micro/nano sized objects and to achieve handling of very small and different in properties objects having specific behavior, respectively. Besides controllable manipulation with different in properties objects, these tools should not affect the examined micro/nano environment, i.e. should be "small and passive" in any sense. That is why a variety of grippers dedicated for different applications have been developed during the recent decade [2], [3]. Despite of the progress, most available microgrippers are still suffering drawbacks

\footnotetext{
${ }^{*}$ Corresponding author.
} 
like: high voltage power supply required, short lifetime, low detection sensitivity and high price. In some cases embedded feed-back sensors are integrated but in general they are non-linear and have low detection sensitivity.

Microgrippers with thermo-mechanical actuation have been considered as one of the promising solutions for bio-medical applications [4]. At the same time, due to the high dissipated power in small-size tools, this type of microgrippers suffers of additional problems like: 1) it is easy to overheat the handled object if non-proper design is used; 2) due to elevated temperatures of operation there is a feed-back sensor parameter drift.

\section{Design Considerations of Thermo-mechanically Driven Monolithic Microgripper}

To overcome the above mentioned obstacles in further development of monomorph compliant microgripper, subject of this paper, following considerations are taken into account:

- in order to manufacture prototypes of a gripper with embedded feed-back sensing, a single-crystal silicon raw material was preferred. A piezoresistive sensing method of arms deflection was selected. Piezoresistors are self-aligned to sidewalls of both gripper's arms and are located close to their fixed ends, where the stress due to deformation reaches it's maximum value. Thus, a maximal sensitivity of the deflection sensing was achieved;

- it was considered as a very important factor to get low voltage durable devices, so electrostatic and piezoelectric actuation options were rejected. Thermo-mechanical actuation was chosen because it has several advantages like: low-voltage, low-power supply, it is step-less and hysteresis-less method, no technology complication due to the actuator integration, etc.

- power on-time and total heat dissipation, respectively, have been reduced via choosing normally closed type of a microgripper. Further, in order to provide simple layout, a design with thermo-actuating elements placed between gripper's arms has been considered for experiments reported;

- since gripper's arms are moving in the plane of substrate, heating element should be designed to avoid off-plane bending. That's why a monomorph actuator element was preferred. In this particular case a highly doped diffusion heater was used for thermo-actuator element. Since the coefficient of thermal expansion of silicon is as low as $4.2 .10^{-6}{ }^{\circ} \mathrm{C}^{-1}$, the length of the heated element should be maximized in order to achieve sufficient end-effector opening range. In the current embodiment the length of the double-folded compliant mechanism is $425 \mu \mathrm{m}$. Furthermore, a specific geometry, providing mechanical amplification of thermally-driven displacement, has been used;

- any overheating of the handled object is further suppressed by placing the monomorph actuator at a maximal distance of gripper jaws and, additionally, griper's arms were designed to have as large as possible surface area for intense heat dissipation. 


\section{Description and Principle of Operation}

Microgripper shown in Fig. 1 consists of a body 1 and two symmetrical arms, 2 and 2 ' respectively, each of them having thickness between $12 \mu \mathrm{m}$ and $15 \mu \mathrm{m}$ and located on the top surface of the body at a distance of $520 \mu \mathrm{m}$ from each other. Arms have also deformable parts 3 and 3' where piezoresistors 4 and 4' are embedded on their sidewalls thus sensing lateral deflections of the arms. The distance between two jaws of the gripper 5 was set to about $10 \mu \mathrm{m}$. The arms have been provided with squareshaped hollows 6 which give extra heat dissipating surface and reduce heat transfer from the body to the jaw's area, where the manipulated object have to be in contact with the gripper. Special care was taken to get stiff arm-construction and to prevent it's bending elsewhere, besides deformable parts 3 and 3', so arm-elements 7 have been added.

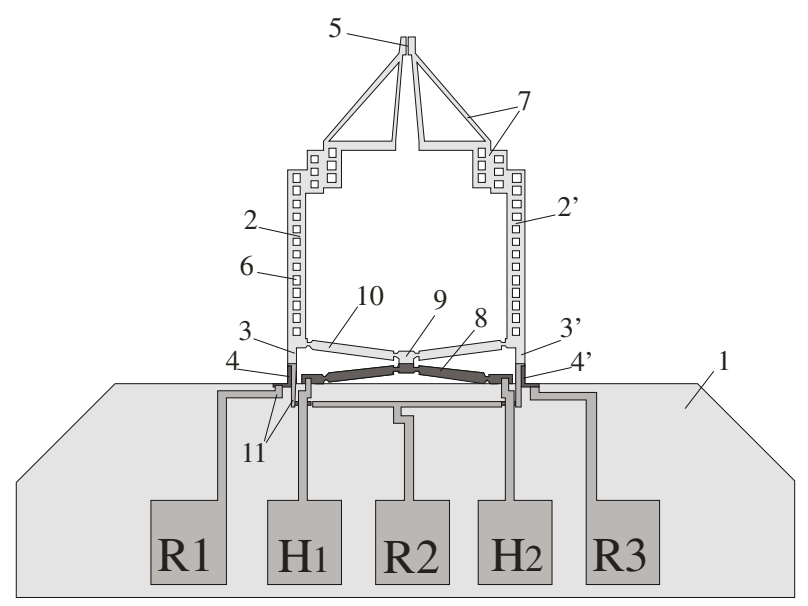

Fig. 1. Schematic layout of thermo-mechanically driven monolithic micro-gripper

When DC current in the range of 5-30 mA is supplied to terminals $\mathrm{H}_{1}$ and $\mathrm{H}_{2}$ of heavily diffusion-doped silicon heating element 8 with resistivity of 200-250 $\Omega$, the Joule heat causes temperature rise. The heated compliant element having $425 \mu \mathrm{m}$ length and sheet resistance of $6 \Omega / \square$, consists of three parts with flexible joins (hinges): two symmetric beams 8 and a central part 9 , with both outer ends of this compliant mechanism being fixed. Due to compliant design, thermal expansion is converted into part 9 translation movement at off-substrate edge direction and its movement is transferred by transmission beams 10 to gripper arms causing jaw's opening. Regulating the heating of the compliant element, one can set the gripper opening at desirable width. Once the power supply is interrupted, due to heat exchange with environmental media, griper comes back to closed position.

Embedded piezoresistors, which are sensitive to in-plane movement of gripper arms, are located into deformable area of the arms' fixed end. These resistors are connected with metal tracks 11 to $\mathrm{R}_{1}, \mathrm{R}_{2}$ and $\mathrm{R}_{3}$ pads. Thus, the end-effector movement causes a changing piezoresistor value. In this way, arm-end position sensing and 
controllable manipulation of the objects could be achieved. The maximum value of the gripping force is defined by the stiffness of the deformable parts 3 and 3 ' of the arms, but a range of intermediate forces is achieved via permanent heating during object handling, as well.

\section{Microgripper Prototype Processing}

The monolithic gripper prototypes have been micro machined by using double-side polished n-type (100) silicon wafers with a resistivity of 4-6 $\Omega . \mathrm{cm}$ and TTV $<2 \mu \mathrm{m}$. Fig. 2 shows a drawing of gripper arm cross-section. Gripper's micromechanical elements are made by means of combination of dry surface and wet bulk micromachining processes as an integral part of recently developed technology for lateral actuated MEMS manufacturing. P-type diffusion resistors 4 and 4' are self-aligned to sidewalls of deformable part 3 of gripper arm. In order to provide piezoresistive properties of diffusion layers, the sheet resistivity was set at range of $250 \pm 20 \Omega / \square$ and the resistors have been oriented in [110] direction. Piezoresistor's non-rectifying contacts are provided by overlapping of it's both ends with heavily doped p+-areas 13 and this structure is completely electrically insulated by silicon dioxide layer 14 . A contact via 15 have been opened through the insulating layer to make available galvanic contact of metal tracks 11 with above mentioned both piezoresistor's ends.

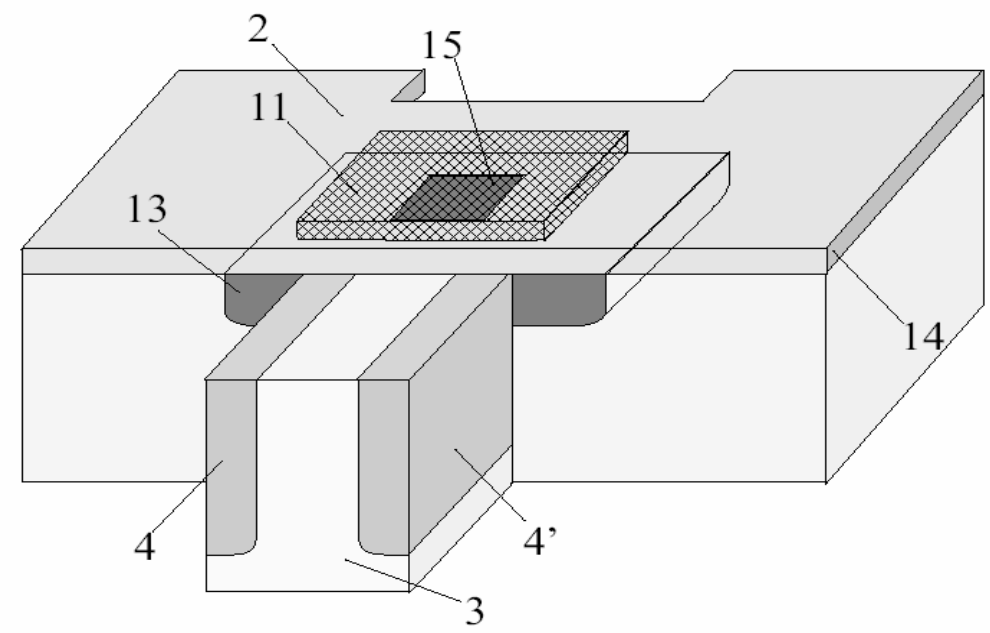

Fig. 2. Cross-section of gripper arm with sidewall embedded piezoresistors on deformable part

\section{Results}

\subsection{Prototypes of Thermo-mechanically Driven Monolithic Microgripper}

Fig. 3 shows a Scanning Electron Microscopy photograph of microgripper prototype which is dedicated to bio-medical applications. The gripper has been implemented as 
a monomorph compliant mechanism and special care was taken for reducing temperature in the area of jaws 5. Despite high temperatures required for thermomechanical actuation, this particular design provides high heat resistance along the grippers' arms and large sidewall area for heat exchange with environmental media. At the same time the design provides sufficient mechanical stiffness of the microgripper arms $2\left(2^{\prime}\right)$.

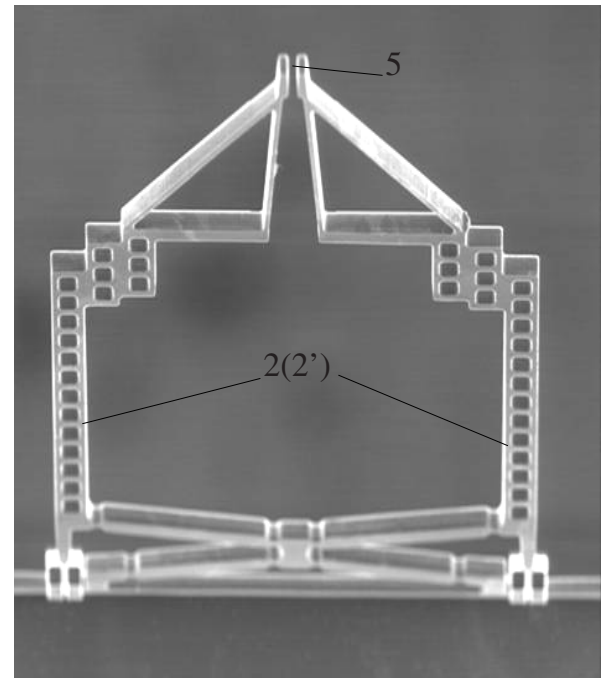

Fig. 3. SEM photo of thermo-mechanically driven monolithic gripper

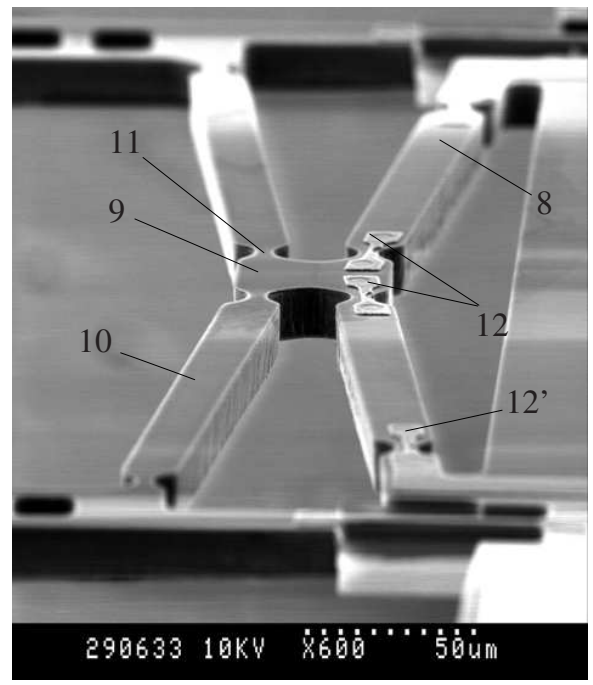

Fig. 4. SEM photo of micro-gripper's compliant mechanism with thermo-mechanical actuator

The scanning electron micrograph (Fig. 4) shows the above mentioned compliant mechanism in more details: right part is the heavily doped heater 8 and left part is the translation transmitting beams 10. Since cross-section area of heating element 8 is changing along it's length, the resistance of the flexible joints (hinges) 11 is higher than resistance in the rest parts of it. In order to avoid overheating in these areas, additional metal shunts: central 12 and outer $12^{\prime}$ are provided. Shunts are made of the same thin film material as metal wiring and for these particular prototypes metal layer was $0.8 \mu \mathrm{m}$ thick aluminum (Al).

\subsection{Electrical Measurements}

To determine the deflection of the gripper's arms, the heater is power supplied by a DC current as shown in Fig. 1. Heating power was determined by measuring the voltage drop over the monolithic heater. 


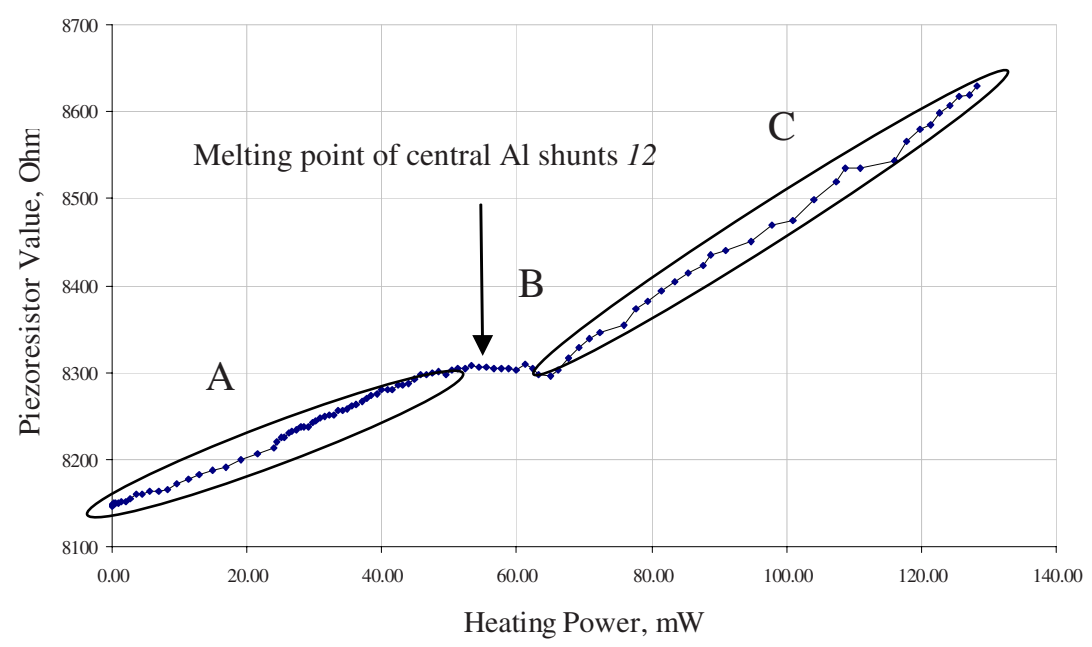

Fig. 5. Piezoresistor value change vs. Heating power

Fig. 5 shows a typical plot of measured piezoresistor values vs. applied heating power. Three distinguished regimes are observed depicted in the three distinctive branches of the curve. The first one (A) is when the heating power is between 0 to approx. $50 \mathrm{~mW}$ which shows a linear increasing resistance. Similar behavior could be observed for the third range (C) between 70 and $140 \mathrm{~mW}$. For the middle part (B) of heating power between 50 and $70 \mathrm{~mW}$ a saturation behavior was found. It was found that latest range corresponds to heating of compliant central part 9 (Fig. 4) up to 660 ${ }^{\circ} \mathrm{C}$, and melting of the central aluminum shunts 12 . Optical microscopy observations confirm this hypothesis but more detailed studies of this region of heating power will be conducted further.

\subsection{Mechanical Behavior of Micro-gripper}

The opened and closed jaws of the gripper are visualized on Figures 6 and 7.

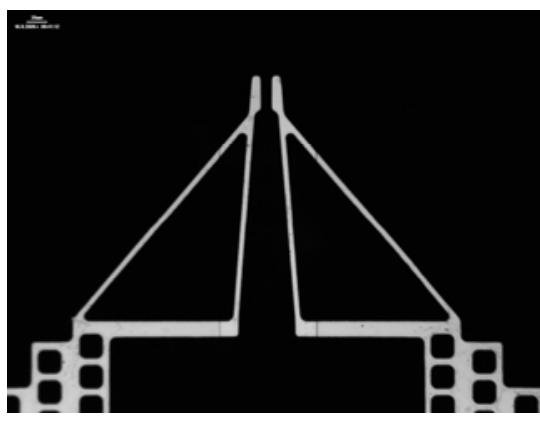

Fig. 6. Photo of closed microgripper jaws

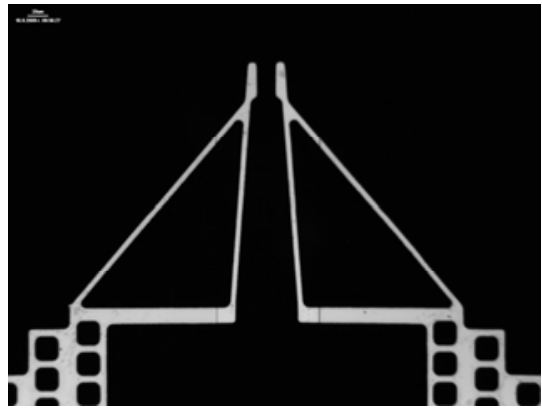

Fig. 7. Photo of opened microgripper jaws 


\section{Thermal Behavior: FE Simulation and Experimental Results}

Experimental measurement of temperature distribution over the monolithic microgripper surface was not available within present study, thus the computer simulation 3D model has been derived and extruded from the photolithography masks. The control equations consist of the steady-state Conductive Media DC (emdc), General Heat Transfer (htgh) and Solid Stress-Strain (smsld) from the Model Navigator tool of COMSOL Multiphysics. The relevant boundary conditions have been selected and adapted to the experimental results shown in Fig. 8. One observes that saturation temperature within the region of gripper's jaws, shown in Fig. 9 could reach $460^{\circ} \mathrm{K}$ for as low heating power as $20 \mathrm{~mW}$. Thus, bio-compatible manipulations $\left(\mathrm{T}<315^{\circ} \mathrm{K}\right)$ with present microgripper are limited to short power on-time cases, only.

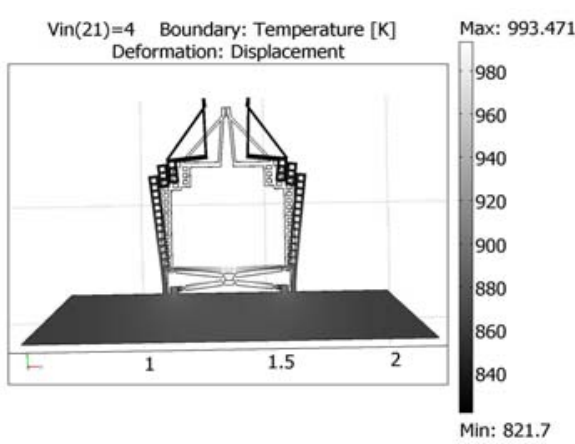

Fig. 8. Temperature distribution over the micro-gripper at typical conditions

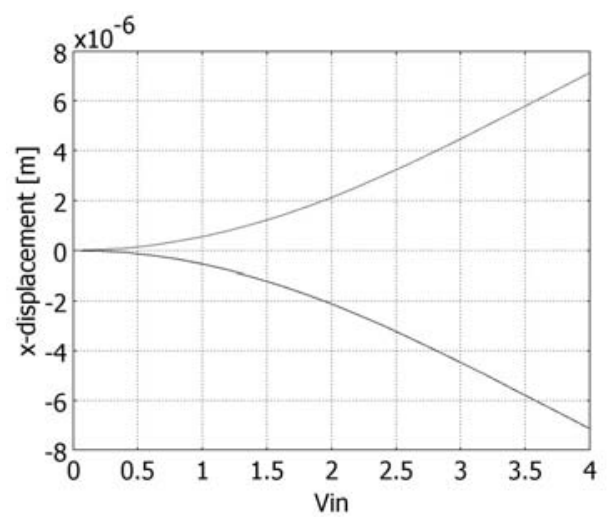

Fig. 10. Micro-gripper jaws opening as a function of applied current (simulation)

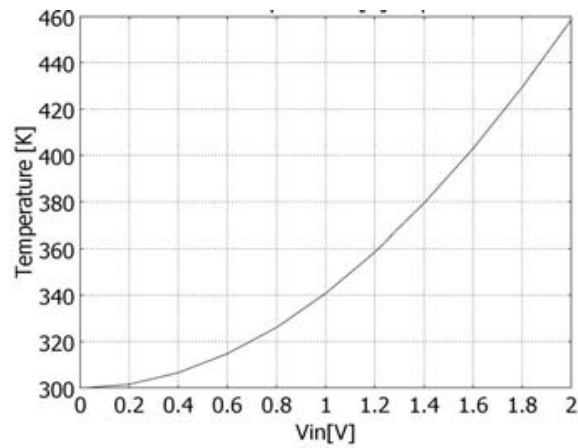

Fig. 9. Micro-gripper jaws' saturation tempe-rature as a function of applied heating power

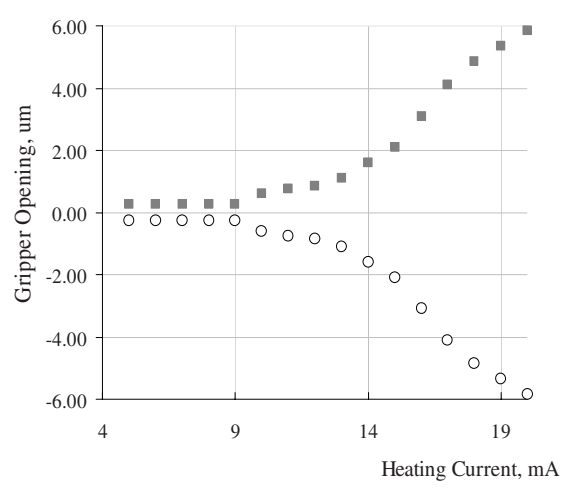

Fig. 11. Micro-gripper arms opening vs. heating current (experimental) 
Figs. 10 and 11 represent a comparison of the computer simulation data for microgripper's arms displacement -- see Fig. 10, with the experimentally measured results shown in Fig. 11. One concludes a good agreement between the values of microgripper jaws opening measured with the values predicted via computer simulation.

Finally, let us stress that the experimentally measured average value of the coefficient between the electrical voltage applied and each arm displacement is $1 \mu \mathrm{m} / \mathrm{V}$, which demonstrates that indeed low voltage operation control of the action of the monolithic thermo-mechanically driven micro gripper has been achieved within the realization suggested.

\section{Conclusion}

In the current article a low voltage monolithic microgripper with thermo-mechanical actuator and piezoresistive deflection feedback has been developed and the results of an initial study of its characteristics have been reported. The experimental results obtained demonstrate a concept proof.

A detailed study of heat transfer dynamics and forces [5] acting between and on the jaws of the gripper are under development in the case when there is or there is no object of a particular shape - spherical, ellipsoidal, etc., between them. This force will depend on the material of the jaws, on the roughness of their surfaces, on the possible inclination between them, on the environmental conditions in which the gripper is working, etc. Further development is also performed aimed to integrate the microgripper suggested into a robotic pick-and-place system for individual cell handling.

Acknowledgements. Authors are thankful to Mr. J. Kirov and Mr. P. Pavlov from AMG Technology Ltd. for processing the prototype and measurement of samples.

The partial financial support of grant TK171/08 of Bulgarian NSF is gratefully acknowledged.

\section{References}

[1] Mayyas, M., Zhang, P., Lee, W.H., Shiakolas, P., Popa, D.: Design Tradeoffs for Electrothermal Microgrippers. In: Proceedings 2007 IEEE International Conference on Robotics and Automation, Roma, Italy, April 10-14 (2007)

[2] Andersen, K.N., Carlson, K., Petersen, D.H., Mølhave, K., Eichhorn, V., Fatikow, S., Bøggild, P.: Electrothermal Microgrippers for Pick-and-place Operations. Microelectronic Engineering 85, 1128-1130 (2008)

[3] Mølhave, K., Hansen, O.: Electro-thermally Actuated Microgrippers with Integrated Force Feedback. Journal of Micromechanics and Microengineering 15, 1265-1270 (2005)

[4] Solano, B.P., Gallant, A.J., Wood, D.: Design and Optimization of a Microgripper: Demonstration of Biomedical Applications Using the Manipulation of Oocytes. In: Proceedings DTIP - 2009, Rome, Italy (2009)

[5] Dantchev, D., Kostadinov, K.: On the Environmental Influence on the Force Between Two Metallic Plates of a Gripper Immersed in a Nonpolar Fluid: the Role of the Temperature and the Chemical Potential. In: Proceedings of the 4M/ICOMM International Conference on Multi-Material Micro Manufacture (4M/ICOMM 2009), Karlsruhe, Germany, September 23-25, pp. 297-300 (2009) 